\title{
Relativistically invariant extension of the de Broglie-Bohm theory of quantum mechanics
}

\author{
Chris Dewdney and George Horton
}

\begin{abstract}
We show that quantum mechanics can be given a Lorentz-invariant realistic interpretation by applying our recently proposed relativistic extension of the de Broglie-Bohm theory to deduce non-locally correlated, Lorentz-invariant individual particle motions for the EPR experiment and the double-interferometer experiment proposed by Horne, Shimony and Zeilinger.
\end{abstract}

PACS numbers: 03.70,03.65 
Relativistically invariant extension of the de Broglie-Bohm theory of quantum mechanics2

Division of Physics, University of Portsmouth. Portsmouth PO1 2DT. England

\section{Introduction}

In the de Broglie-Bohm approach to quantum theory configuration space is the fundamental arena. In non-relativistic quantum theory, given the wave function of an $n$-particle system $\Psi\left(\vec{r}^{(1)}, \vec{r}^{(2)} \ldots . \vec{r}^{(n)} ; t\right)$, where the bracketed superscript labels the particle, and some "initial" point in the configuration space-time, the entire motion of the system in space and time is determined. Each initial point in the configuration space-time determines one possible motion of the system, but, of course, we cannot control which trajectory actually occurs in a given case. With entangled wave functions the velocity of a given particle at a particular point will depend on the positions of all the other particles in the system. Naturally, in a non-relativistic theory one considers the positions of the particles at the same time. However, in relativistic, multi-time quantum theory there is no universal time coordinate and in a given inertial reference frame, $\Sigma$, the argument of the wave function may be written $\left(\vec{r}^{(1)}, t^{(1)} \ldots . \vec{r}^{(n)}, t^{(n)}\right)$, using an appropriate coordinate system. Just as in the non-relativistic theory, to obtain a definite trajectory for the relativistic system an "initial" point in the configuration space-time must be specified. Also like the nonrelativistic case, we cannot control which initial point is the actual point in a given run of an experiment. However, as we have discussed in detail elsewhere ([1, 2]), and unlike the non-relativistic case, the specification of this point alone, although necessary, is not sufficient to determine a unique configuration space-time trajectory of the system. The problem is that with entangled wave functions the individual particle velocities depend on all the arguments of the wave function; so, in addition to their initial values, one must specify a rule which coordinates the individual arguments in the wave function beyond their initial coordinated values, in order to integrate the equations of motion. It is important to be clear that the coordination that we are discussing is not the coordination of points on given, pre-existing individual particle world lines. The particle world lines are not specifiable at all unless one has decided how the particle coordinates are to be coordinated beyond the initial chosen point. The choice of the initial point is arbitrary, just as as in the non-relativistic theory but, just as in the non-relativistic theory, each choice can be associated with a certain probability density. In general there will be some set of points which corresponds with the particular experimental set up being considered. Different choices of initial point, $\left(\vec{r}^{(1)}, t^{(1)} \ldots \vec{r}^{(n)}, t^{(n)}\right)$, will lead to different trajectories. Furthermore, even given the same initial point, different rules of coordination of the particle coordinates will lead to different configuration space-time trajectories.

Several suggestions have been made concerning the coordination of the particle coordinates. For example, Bohm and Hiley [3] have proposed that there exists a preferred frame of reference in which one takes equal time steps for each particle's time coordinate in integrating the equations of motion. In Bohm's approach relativistic invariance appears only as a property of the statistical results of measurement whilst 
the individual processes themselves are not invariant. Suarez 4] has proposed a "multisimultaneity" theory in which the measuring devices determine the coordination of the particles along their simultaneity hyperplanes, but this theory has experimental consequences different to quantum mechanics and has in fact been refuted [5]. Other approaches [6, [7], 8], although based on four-velocities, have used an arbitrary foliation of space-time to provide a basis for integrating the equations of motion; but then the particle trajectories for given initial conditions, calculated using different foliations are not lorentz transforms of each other. To the extent that the positions and momenta depend on the choice of foliation a theory cannot be considered a theory of "beables".

Our approach [1] is based on the existence of time-like four velocities, for both bosons and fermions, and uses a relativistically invariant rule, utilizing the invariant light-cone structure, to produce the system trajectory. In our approach the coordination of the coordinates is achieved by advancing the arguments in the wave function so that, for all $n$

$$
\left(\Delta t^{(n)}\right)^{2}-\left(\Delta x^{(n)}\right)^{2}-\left(\Delta y^{(n)}\right)^{2}-\left(\Delta z^{(n)}\right)^{2}=\Delta \tau^{2}
$$

Our approach yields a unique and relativistically invariant trajectory in the configuration space-time of the system. The configuration space-time trajectory determines both the individual world lines of the particles and the coordination of the points on the individual particle world lines. The motion is irreducibly defined in the configuration space-time spanned by $\left(\vec{r}^{(1)}, t^{(1)} \ldots \vec{r}^{(n)}, t^{(n)}\right)$ and a line in this space determines the sets of values of the coordinates that are coordinated (taken together in the calculation of the trajectory). The system's motion is determined once an "initial" point in the configuration space-time is specified and our use of the word "initial" merely indicates a starting point for the calculation. A particular experiment will be consistent with some set of initial points, distributed with the appropriate density in the configuration spacetime, which determine the possible motions of the system. Initial points, not within the appropriate set in configuration space-time may be chosen but, in general, these will correspond to a different experiment.

Just as in non-relativistic de Broglie-Bohm theory there is no wave-packet collapse in our relativistic extension. Measurements play no fundamental role, they are simply interactions between systems during which a correlation is introduced between their variables such that by observing one variable one can infer the value of the other. The inclusion of the additional system requires the enlargement of the configuration spacetime in which the measurement must be described as a dynamical process relating system and measuring device coordinates.

Since the system trajectory in configuration space-time is calculated in a relativistically invariant way it cannot matter which frame of reference we choose merely to describe the system's motion; changing frames of reference simply amounts to a passive re-assignment of coordinates. The outcome of any specific experiment, for a given "initial" point in the configuration space-time, will be the same in all frames. If, in a given experiment, parts of the apparatus are in relative motion (such as the beam 
splitters in the experiment of Stefanov et al $[\underline{5}$ ) it cannot matter whether we choose the rest frame of one moving part or that of another, the system's motion, for a given initial point in configuration space-time, will be the same.

In the following section we consider how our approach can be applied to the case of spin entanglement in an EPR experiment. We then go on to illustrate our approach in the context of the two-particle experiment proposed by Horne et al. [9].

\section{EPR experiment}

Consider the Einstein-Podolsky-Rosen (EPR) experiment, in the form first proposed by David Bohm, in which two (space-like separated) spin-one-half particles are entangled in a total spin zero state. The spinor wave function, in an inertial frame $\Sigma$ and using an appropriate coordinate system, may be written

$$
f_{1}\left(\vec{r}^{(1)}, t^{(1)}\right) f_{2}\left(\vec{r}^{(2)}, t^{(2)}\right)\left[\left(\begin{array}{l}
1 \\
0
\end{array}\right)^{(1)}\left(\begin{array}{l}
0 \\
1
\end{array}\right)^{(2)}-\left(\begin{array}{l}
0 \\
1
\end{array}\right)^{(1)}\left(\begin{array}{l}
1 \\
0
\end{array}\right)^{(2)}\right]
$$

where $f_{1}$ and $f_{2}$ are localized wave packets moving in opposite directions along the $x$ axis and the bracketed superscript labels the particles. Figure 1 shows the particle paths in a space-time diagram of the experiment in the $\Sigma$ frame. At time $t_{i}$, in $\Sigma$, the $z$ components of the spins of the particles are measured by Stern-Gerlach apparatuses yielding the wave function, for both $t^{(2)}$ and $t^{(1)}>t_{i}$.

$$
\left[f_{1}^{+}\left(\vec{r}^{(1)}, t^{(1)}\right) f_{2}^{-}\left(\vec{r}^{(2)}, t^{(2)}\right)\left(\begin{array}{l}
1 \\
0
\end{array}\right)^{(1)}\left(\begin{array}{l}
0 \\
1
\end{array}\right)^{(2)}-f_{1}^{-}\left(\vec{r}^{(1)}, t^{(1)}\right) f_{2}^{+}\left(\vec{r}^{(2)}, t^{(2)}\right)\left(\begin{array}{l}
0 \\
1
\end{array}\right)^{(1)}\right.
$$

where $f^{+}$and $f^{-}$are wave packets moving additionally with opposite momenta along the direction of the Stern Gerlach fields. In order to calculate trajectories and spin-vector orientations for the particles one needs a definition of the velocity and spin as beables and, as we have already emphasized, a rule for coordinating the system's coordinates. A hidden-variable theory for the Dirac equation was provided by Bohm in 1953 [10]. In order to begin the calculation of the trajectory of the system in its eight-dimensional configuration space-time one needs to choose an initial position $\left({\overrightarrow{r_{0}}}^{(1)}, t_{0}^{(1)}, \vec{r}_{0}^{(2)}, t_{0}^{(2)}\right)$. Then, given this initial coordination of the particle coordinates, our Lorentz-invariant rule can be applied to calculate the configuration space-time trajectory and associated individual-particle spin components. The result of the calculation can be displayed in a "look-up" table as follows:

$$
\begin{array}{cccccc}
\vec{r}_{0}^{(1)} & t_{0}^{(1)} & \vec{s}_{0}^{(1)} & \vec{r}_{0}^{(2)} & t_{0}^{(2)} & \vec{s}_{0}^{(2)} \\
\vec{r}_{1}^{(1)} & t_{1}^{(1)} & \vec{s}_{1}^{(1)} & \vec{r}_{1}^{(2)} & t_{1}^{(2)} & \vec{s}_{1}^{(2)} \\
\vec{r}_{2}^{(1)} & t_{2}^{(1)} & \vec{s}_{2}^{(1)} & \vec{r}_{2}^{(2)} & t_{2}^{(2)} & \vec{s}_{2}^{(2)} \\
. . & . . & . . & . . & . . & . . \\
\vec{r}_{n}^{(1)} & t_{n}^{(1)} & \vec{s}_{n}^{(1)} & \vec{r}_{n}^{(2)} & t_{n}^{(2)} & \vec{s}_{n}^{(2)}
\end{array}
$$


Each row of the table lists a set of coordinated coordinates and locates a point on the configuration space-time trajectory of the system. Taking the two halves of the table separately, corresponding to each of the individual particle coordinates:

$$
\begin{array}{ccc}
\vec{r}_{0}^{(1)} & t_{0}^{(1)} & \vec{s}_{0}^{(1)} \\
\vec{r}_{1}^{(1)} & t_{1}^{(1)} & \vec{s}_{1}^{(1)} \\
\vec{r}_{2}^{(1)} & t_{2}^{(1)} & \vec{s}_{2}^{(1)} \\
\quad . . & . . & . . \\
\vec{r}_{n}^{(1)} & t_{n}^{(1)} & \vec{s}_{n}^{(1)}
\end{array}
$$

and

$$
\begin{array}{ccc}
\vec{r}_{0}^{(2)} & t_{0}^{(2)} & \vec{s}_{0}^{(2)} \\
\vec{r}_{1}^{(2)} & t_{1}^{(2)} & \vec{s}_{1}^{(2)} \\
\vec{r}_{2}^{(2)} & t_{2}^{(2)} & \vec{s}_{2}^{(2)} \\
\quad . . & . . & . . \\
\vec{r}_{n}^{(2)} & t_{n}^{(2)} & \vec{s}_{n}^{(2)}
\end{array}
$$

yields world-lines and associated spin-vectors for each particle, but in taking the coordinates separately one looses the information regarding the coordination of the coordinates present in the configuration space-time trajectory. Nonetheless, each position on the world line of each particle has a definite spin associated with it, combinations of individual particle coordinates from the same row of the full table will have total spin zero, but combinations from different rows need not.

Let us consider an example. If the initial point in configuration space-time is chosen such that $t_{0}^{(1)}=t_{0}^{(2)}<<t_{i}$, in $\Sigma$, then for some specific choice of $r^{(1)}$ and $r^{(2)}$, a configuration space-time trajectory and particle world lines, can be calculated. For this specific choice of the initial time coordinates the particles will reach the Stern Gerlach devices at more or less the same time in $\Sigma$. The "look-up" table will then be as shown in the following table:

$$
\begin{array}{cccccc}
\vec{r}_{0}^{(1)} & t_{0}^{(1)} & (0,0,0) & \vec{r}_{0}^{(2)} & t_{0}^{(2)} & (0,0,0) \\
\vec{r}_{1}^{(1)} & t_{1}^{(1)} & (0,0,0) & \vec{r}_{1}^{(2)} & t_{1}^{(2)} & (0,0,0) \\
\vec{r}_{i}^{(1)} & t_{i}^{(1)} & (0,0,+1) & \vec{r}_{i}^{(2)} & t_{i}^{(2)} & (0,0,-1) \\
. . & . . & . . & . . & . . & . . \\
\vec{r}_{n}^{(1)} & t_{n}^{(1)} & (0,0,+1) & \vec{r}_{n}^{(2)} & t_{n}^{(2)} & (0,0,-1)
\end{array}
$$

Now if one chooses to compare values at the uncoordinated times $t^{(2)}=t_{k}>t_{i}$ but $t^{(1)}=t_{j}<t_{i}$, one finds that particle two has a definite spin in the minus $z$ direction but particle one has spin zero. However, there is no contradiction in this as no measurement of the spin of particle one has taken place; if such a measurement were to be carried out one would not find the value zero, but \pm 1 , and this would obviously correspond to a different experiment in which the configuration space trajectory would have to be calculated afresh. (This is similar to the situation in non-relativistic de Broglie-Bohm theory wherein the values assigned to variables are not in general the eigenvalues of the associated operators unless a measurement process is carried out.) In general there 
will be some inertial observer whose frame of reference will correspond with $t_{k}^{\prime(2)}=t_{j}^{\prime(1)}$, and this observer may be tempted to assume that the particle coordinates must be coordinated on these special equal-time hyperplanes, but this would be to adopt a frame-dependent and hence non-relativistic rule. In our approach the coordination of the particle coordinates has the status of a beable, just as the positions of the particles do in the non-relativistic de Broglie-Bohm theory. Hence for a given run of an experiment the coordination is fixed and is no more an arbitrary choice of an observer than the positions of the particles are in the non-relativistic theory. For the purposes of calculating the possible motions of the system one may choose a variety of initial coordinated coordinates, just as one may choose a variety of initial positions in the non-relativistic de Broglie-Bohm theory. However, just as in the choice of initial coordinates in the non-relativistic theory, there will be some subset of all possible choices for $\left({\overrightarrow{r_{0}}}^{(1)}, t_{0}^{(1)}, \vec{r}_{0}^{(2)}, t_{0}^{(2)}\right)$ that in fact correspond with a non-negligible probability for a given experimental design.

Since our method of calculation is lorentz invariant, representation of the configuration-space-time trajectory using another inertial frame of reference involves only a simple passive transformation of the coordinated coordinates in the look-up table, hence for a given initial position in configuration space-time the outcome of the experiment is the same in all inertial frames. In a given run of the experiment there will be an unknown (and uncontrollable) configuration space-time trajectory, L, which describes the actual evolution of the system and the manner in which the particle coordinates are coordinated. If a different initial point in configuration space-time, not lying on $\mathrm{L}$ is chosen, then the coordination of the coordinates is different, leading to a different configuration space-time trajectory and there should be no surprise if this leads to a different and apparently "contradictory" outcome of the experiment. The theory is deterministic in the configuration space-time in which the trajectories do not cross. Any point lying on L, if chosen as the starting point for the calculation will give rise to the same $\mathrm{L}$ and the same trajectories in space-time.

The assumption that the coordination of the particle coordinates is to be arbitrarily decided, in a non-lorentz-invariant way, according to the equal time surfaces of the inertial frame chosen to describe the experiment, is the origin of the so-called problems associated with making hidden-variable theories of quantum mechanics lorentz invariant at the level of the hidden variables. Hardy [11] implicitly applies such a non-lorentz invariant rule to demonstrate the impossibility of lorentz-invariant hidden variables. But it should be clear that choosing a non-lorentz invariant rule to determine the coordination of the particles' coordinates will have non-lorentz invariant consequences.

\section{The Horne, Shimony and Zeilinger experiment}

We have chosen this experiment since a similar, double-interferometer experiment was used by Hardy [11] to support his argument that hidden-variable theories of relativistic quantum theory must have non-lorentz-invariant hidden variables. Hardy's experiment 
involves pair annihilation, a feature that we would prefer to avoid, since his argument can be discussed without this complication.

Consider the two-particle interferometer illustrated in figure 2, discussed first by Horne, Shimony and Zeilinger [9] and in the context of the non-relativistic de BroglieBohm theory by Dewdney and Lam [12. At $t=0$, in the $\Sigma$ frame, the source $S$ emits two distinguishable non - interacting particles, 1 and 2 into the spatially distinct paths $a, b, c$ and $d$. These paths are associated with the single - particle localized wave packets $a^{(1)}, b^{(2)}, c^{(2)}$ and $d^{(1)}$ respectively where the bracketed superscripts 1 and 2 label the particles. The particles are emitted so that either:

(i) particle 1 follows path $a$ and particle 2 follows path $c$ or,

(ii) particle 1 follows path $d$ and particle 2 follows path $b$.

Hence, in $\Sigma$, the wave function on the $t=0$ hypersurface can be taken to be

$$
\Psi\left(\vec{r}^{(1)}, 0 ; \vec{r}^{(2)}, 0\right)=\frac{1}{\sqrt{2}}\left[a\left(\vec{r}^{(1)}, 0\right) c\left(\vec{r}^{(2)}, 0\right)+d\left(\vec{r}^{(1)}, 0\right) b\left(\vec{r}^{(2)}, 0\right)\right]
$$

The particle on path $a$ experiences a variable phase shift $\phi$, whilst the other, on path $b$, receives a variable shift $\chi$. After reflection at the mirrors, each particle encounters a beam-splitter $\left(H_{1}\right.$ or $H_{2}$ ) through which it may be transmitted or reflected with equal probability, regardless of the settings of the phase shifters. Each particle eventually emerges either in the positive or the negative sense of the vertical axis, which is designated $z$. The outcomes for particle 1 will be referred to as $1^{+}$or $1^{-}$, and similarly $2^{+}$or $2^{-}$for particle 2 . There are no single particle interferences, if one just looks where particle 1 emerges the probabilities of $1^{+}$and $1^{-}$are both equal to 0.5 independently of the settings of the phase shifters. The same is true of particle 2. However, the joint probabilities show interference effects as they do depend on the settings of the phase shifters as follows:

$$
\begin{aligned}
& P\left\{1^{+} 2^{+}\right\}=\frac{1}{4}(1+\cos (\chi-\phi)) \\
& P\left\{1^{+} 2^{-}\right\}=\frac{1}{4}(1-\cos (\chi-\phi)) \\
& P\left\{1^{-} 2^{+}\right\}=\frac{1}{4}(1-\cos (\chi-\phi)) \\
& P\left\{1^{-} 2-\right\}=\frac{1}{4}(1+\cos (\chi-\phi))
\end{aligned}
$$

\subsection{The configuration space-time model}

The critical aspects of the motion of the particles take place in the one dimension $z$, perpendicular to the beam-splitters; so all features of the evolution of the wave function, relevant to this discussion, take place in the four-dimensional configuration space-time $\left(z^{(1)}, t^{(1)} ; z^{(2)}, t^{(2)}\right)$. Only the final scattering from the beam-splitters $\left(H_{1}\right.$ and $\left.H_{2}\right)$ need be modelled in detail as the full reflections (at $M_{1}$ and $M_{2}$ ) simply serve to change the direction of the particles. In figure 3, we illustrate the possible paths of the particles, 
in $\Sigma$, in a space-time $(x, z, t)$ diagram. In this frame the particles scatter from the full reflecting mirrors at $t_{\lambda}$, reach the final beam splitters at $t_{\mu}$ and emerge beyond the interferometers at $t_{\nu}$. The time-dependence of the two packets in configuration spacetime, represented by the two terms in (10), are given in this model by

$$
\Psi\left(z^{(1)}, t^{(1)} ; z^{(2)}, t^{(2)}\right)=\frac{1}{\sqrt{2}}\left[a\left(z^{(1)}, t^{(1)}\right) c\left(z^{(2)}, t^{(2)}\right) e^{i \phi}+d\left(z^{(1)}, t^{(1)}\right) b\left(z^{(2)}, t^{(2)}\right) e^{i \chi}\right]
$$

According to the wave function given in equation (6) the point $\left(z_{0}^{(1)}, t_{0}^{(1)} ; z_{0}^{(2)}, t_{0}^{(2)}\right)$ from which we start calculating the trajectory can be chosen arbitrarily. Let us consider two different scenarios.

3.1.1. Scenario 1: configuration space-time trajectories initiated on $\left(z_{0}^{(1)}, t_{0}^{(1)}=t_{\mu} ; z_{0}^{(2)}, t_{0}^{(2)}=t_{\lambda}\right)$ In this scenario the particles' time coordinates are such that particle 1 at the final beamsplitter is coordinated with particle 2 at the full reflecting mirror. $\left|\Psi\left(z_{0}^{(1)}, t_{\mu} ; z_{0}^{(2)}, t_{\lambda}\right)\right|^{2}$ is shown in figure 4 , which also illustrates, in the reduced configuration space defined by $\left(z^{(1)}, z^{(2)}\right)$, the initial single-particle marginal distributions and the general character of the particle trajectories. For this scenario, the main characteristics of the particles' motions can be deduced from the fact that configuration space trajectories do not cross. Integrating the equations of motion from specific choices for $z^{(1)}$ and $z^{(2)}$ it is clear that when particle 1 reaches its beam splitter, the packets for particle 2 are still well separated and effectively non-overlapping. Consequently, the two packets in configuration space are non overlapping and hence behave independently as particle 1 scatters from its beam splitter. In a single instance the point representing the configuration of the system must lie in one or other of the configuration space wave packets. If the coordinate of particle 2 lies in $c$ then the effective configuration space wave function is

$$
a\left(z^{(1)}, t_{\mu}\right) c\left(z^{(2)}, t_{\lambda}\right) e^{i \phi}
$$

a simple product. Whereas if the coordinate of particle 2 lies in $b$ then the effective wave function is

$$
d\left(z^{(1)}, t_{\mu}\right) b\left(z^{(2)}, t_{\lambda}\right) e^{i \chi}
$$

In this scenario the possible trajectories of particle 1 at its beam splitter are just those of a single particle scattering from a beam splitter. The non-crossing of the trajectories now applies to the individual trajectories for particle 1 without reference to the position of particle 2 (save to locate the system in one of the quadrants of the configuration space). If particle 1 is located in the forward part of $a\left(z^{(1)}, t_{\mu}\right)$ it is transmitted and if in the trailing part it is reflected [13]. A similar argument clearly holds for the case in which particle 2 is in $b$, but then we are concerned with the other packet in configuration space.

After particle 1 has scattered there will be four non-overlapping packets in configuration space. Particle 1 is leaving the interferometer whilst particle 2 is 
approaching its beam splitter. The wave function can be represented by

$$
\Psi\left(z^{(1)}, t_{\nu} ; z^{(2)}, t_{\mu}\right)=\frac{1}{2}\left[\begin{array}{c}
\left\{a_{r}\left(z^{(1)}, t_{\nu}\right) e^{-i \frac{\pi}{2}}+a_{t}\left(z^{(1)}, t_{\nu}\right)\right\} c\left(z^{(2)}, t_{\mu}\right) e^{i \phi} \\
+\left\{d_{r}\left(z^{(1)}, t_{\nu}\right) e^{-i \frac{\pi}{2}}+d_{t}\left(z^{(1)}, t_{\nu}\right)\right\} b\left(z^{(2)}, t_{\mu}\right) e^{i \chi}
\end{array}\right]
$$

where the subscripts $r$ and $t$ represent, respectively, the reflected and transmitted parts of the packets $a$ and $d$. As particle 2 progresses towards its beam splitter the four wave packets interfere at the $z^{(2)}$ beam splitter in pairs. The functional form of $a_{r}\left(z^{(1)}, t^{(1)}\right)$ and $d_{t}\left(z^{(1)}, t^{(1)}\right)$ is identical (the reflected part of $a$ coincides with the transmitted part of $d$ ) and we represent this common function by $a_{r} d_{t}$. The same is true of $a_{t}\left(z^{(1)}, t^{(1)}\right)$ and $d_{r}\left(z^{(1)}, t^{(1)}\right)$ which we represent by $a_{t} d_{r}$. Rearranging equation (9) we find

$$
\Psi\left(z^{(1)}, t_{\nu} ; z^{(2)}, t_{\mu}\right)=\frac{1}{2}\left[\begin{array}{c}
a_{r} d_{t}\left(z^{(1)}, t_{\nu}\right)\left\{c\left(z^{(2)}, t_{\mu}\right) e^{-i \frac{\pi}{2}} e^{i \phi}+b\left(z^{(2)}, t_{\mu}\right) e^{i \chi}\right\} \\
+a_{t} d_{r}\left(z^{(1)}, t_{\nu}\right)\left\{c\left(z^{(2)}, t_{\mu}\right) e^{i \phi}+b\left(z^{(2)}, t_{\mu}\right) e^{-i \frac{\pi}{2}} e^{i \chi}\right\}
\end{array}\right]
$$

now the particle 1 wave packets $a_{r} d_{t}$ and $a_{t} d_{r}$ are non-overlapping and so once more each part of the wave function behaves independently. As is clear from equation (10), the fate of particle 2 depends on which packet particle 1 is in and on the relative phase of the packets $c$ and $b$. Notice that as particle 2 approaches its beam-splitter the relative phase of the particle-2 packets $c$ and $b$ depends not just on the phase shift $\chi$ applied to particle-2 packet $b$ but also on the phase shift $\phi$ applied to particle 1 at an arbitrarily distant location. If, in a particular case, particle 1 is in $a_{r} d_{t}$ (in the positive domain of the $z^{(1)}$ axis corresponding with the outcome $1^{+}$) and $\chi-\phi=0$, then all trajectories from $c(2)$ are transmitted and all those from $b(2)$ are reflected. These are the reduced configuration space, $\left(z^{(1)}, z^{(2)}\right)$, trajectories shown in figure 4 in the region where $z^{(1)}$ is positive (on the right of the diagram); they correspond with the outcome $1^{+} 2^{+}$. If on the other hand particle 1 is located in $a_{t} d_{r}$ (in the negative domain of the $z^{(1)}$ axis corresponding with the outcome $1^{-}$) and $\chi-\phi=0$, then all trajectories from $c(2)$ are reflected and all those from $b(2)$ are transmitted. These trajectories are also shown in figure 4 in the region where $z^{(1)}$ is negative (on the left of the diagram); they correspond with the outcome $1^{-} 2^{-}$. Changing the phase difference, $\chi-\phi$, will produce different trajectories but we need not consider the alternatives here.

3.1.2. Scenario 2: configuration space-time trajectories are initiated on $\left(z_{0}^{(1)}, t_{0}^{(1)}=t_{\lambda} ; z_{0}^{(2)}, t_{0}^{(2)}=t_{\mu}\right) \quad$ In this scenario the particles' time coordinates are such that particle 2 at the final beam-splitter is coordinated with particle 1 at the full reflecting mirror. $\left|\Psi\left(z_{0}^{(1)}, t_{\lambda} ; z_{0}^{(2)}, t_{\mu}\right)\right|^{2}$ is plotted in the reduced configuration space $\left(z^{(1)}, z^{(2)}\right)$ corresponding with $t_{0}^{(1)}=t_{\lambda}, t_{0}^{(2)}=t_{\mu}$ in figure 5 , along with indicative trajectories for the case in which $\chi-\phi=0$. The trajectories are deduced in the same manner as for the alternative scenario discussed above and the motion of particle 2 at its beam splitter is independent of the location of particle 1 within the appropriate configuration space packet. Particles in the forward parts of the packets $b(2)$ and $c(2)$ are transmitted whilst those in the trailing parts are reflected. The fate of particle 1 at its beam splitter then depends on the position of particle 2 after passage through the beam splitter. 
3.1.3. Comparison of the scenarios Consider the case in which particle 1 is located in the front part of packet $a(1)$, in scenario 1 this particle will definitely be transmitted (irrespective of the position of particle 2 in its packet), whereas in scenario 2, if particle 2 is in the rear part of $c(2)$, then particle 1 will be reflected. If in a given single run of the experiment the choice between the different scenarios is an arbitrary choice of the "observer" (rather than a "beable" determined by the hidden-variable theory) then there is a contradiction: this is what happens in the multi-simultaneity theory and forms the basis of Hardy's argument. Hardy in fact gives no explicit rule for coordinating the particle time coordinates, but implicitly relies on the rule that the coordinates are to be coordinated along the equal-time hypersurfaces of the arbitrary frame of reference chosen to describe the experiment. This is similar to multi-simultaneity theory which also relies on a non-Lorentz invariant rule.

According to multi-simultaneity theory, the frame of reference in which the two particles are coordinated along the equal time hypersurfaces is chosen to be the rest frame of the massive apparatuses with which the particles interact. The apparatus components in the two distant wings of the HSZ experiment can be put in such a state of motion that, according to multisimultaneity, both of the scenarios that we have discussed apply at once. It is then conjectured that this clash is reflected in nature by the loss of correlations.

Bohm's preferred-frame theory rejects the joint applicability of the two scenarios and argues that there is only one preferred frame, $\Sigma^{\prime}$, in which the results can be calculated correctly. The preferred frame of reference may, or may not, coincide with a frame which has $t_{\lambda}^{\prime(1)}=t_{\mu}^{\prime(2)}$, but at most only one of the scenarios we can calculate can describe the correct motion. The preferred frame is undetectable experimentally.

In our approach the different possible motions for the particles in the two scenarios arise, not as a result of different arbitrary choices of experimenters, but from different initial conditions, $\left(z_{0}^{(1)}, t_{0}^{(1)} ; z_{0}^{(2)}, t_{0}^{(2)}\right)$, that may, or may not, have been physically realized for a given experimental situation and, given the initial values (which determines the coordination of the trajectories) our calculation yields the same motion regardless of our choice of frame of reference. The two scenarios correspond to different and exclusive "experimental runs"; they are not equally valid descriptions of one and the same experimental run. If, for example, the wave packets in fact emerge simultaneously in a given frame of reference, then this determines the ensemble of possible, physically realizable, initial configuration space-time points (and hence coordinations) for this particular experiment. Observers in frames of reference in relative motion will judge simultaneity differently, but this has nothing to do with the actual set of initial points in configuration space-time that are consistent with the experimental conditions or the way in which the points on the individual particle trajectories are in fact coordinated according to our lorentz invariant rule. So, a given instance of the experiment will have a corresponding lorentz-invariant trajectory in the configuration space-time, but, of course, we can neither know in advance which trajectory is actualized nor control the hidden variables. Detection of the particles at the space-time points $\left(z^{(1)}, t^{(1)}\right),\left(z^{(2)}, t^{(2)}\right)$ 
does not reveal sufficient information to determine the configuration-space trajectory which the particles in fact followed; additionally one needs to know whether the particle trajectories were coordinated at these points. In other words, the particles may have been detected at $\left(z^{(1)}, t^{(1)}\right)\left(z^{(2)}, t^{(2)}\right)$ but this does not reveal whether these points are coordinated. Initiating a configuration-space-time trajectory from the point $\left(z^{(1)}, t^{(1)} ; z^{(2)}, t^{(2)}\right)$, with the idea of retrodicting the system trajectory, would assume that the individual trajectories are coordinated by this point, which need not be the case.

\section{Conclusion}

The apparent lack of a covariant extension of the de Broglie-Bohm hidden-variable theory of relativistic quantum mechanics, which respects lorentz invariance at the level of the hidden variables, has often been cited as a major problem. In this paper we have shown how our lorentz-invariant extension of the de Broglie-Bohm approach can be applied to EPR - type experiments to give a unique and unambiguous lorentz invariant description of the individual processes which underlie the statistical predictions of quantum theory. Our approach is in accord with non-relativistic de Broglie-Bohm theory in which, given a system's wave function, one only needs to specify an initial position in the system's configuration space-time in order to completely determine the motion. However, in the relativistic theory the specification of the initial position is no longer sufficient, one also needs to specify a rule according to which the particles' coordinates are coordinated (beyond the initial point) in integrating the equations of motion. The initial point, plus the rule, leads to a unique trajectory in the system's configuration space-time for each experimental run. Hence for any experiment one has a pair of unique world lines along which the coordinates are coordinated according to the given configuration space-time trajectory. One can then choose to describe the welldefined configuration space-time trajectory using any inertial frame that one likes, this choice is arbitrary just as one would expect in a relativistic theory.

The adoption of non-lorentz invariant (or inertial frame dependent) rules of coordination leads to contradictions, as Hardy has demonstrated and as is clear in multisimultaneity theory. In this paper we have shown how the ambiguities discussed by Hardy (and others) can be avoided in our approach by the use of a natural lorentz invariant theory and how the fundamental notion of lorentz invariant "beables" can be retained.

[1] Horton G and Dewdney C 2001 J.Phys.A:Math.Gen 349871

[2] Horton G and Dewdney C 2000 J.Phys.A:Math.Gen 337337

[3] Bohm D and Hiley B J 1994 The Undivided Universe (London: Routledge)

[4] Suarez A and Scarani V quant-ph/9704038

[5] Stefanov A et al. quant-ph/0110117

[6] Durr D, Goldstein S, Berndl K M and Zanghi N 1999 Phys Rev A 602729

[7] Ghose P, Majumdar A S, Guha S and Sau J 2001 Phys Lett A 290205

[8] Holland P R 1999 Phys Rev A 604326

[9] Horne M A, Shimony A and Zeilinger A 1989 Phys. Rev. Lett. 622209 
Relativistically invariant extension of the de Broglie-Bohm theory of quantum mechanics12

[10] Bohm D 1953 Prog. Theor. Phys. 9273

[11] Hardy L 1992 Phys. Rev. Lett. 682981

[12] Dewdney C and Lam M. M. 1990 Phys Lett A 150127

[13] Dewdney C and Hiley B J 1982 Found. Phys 1227

\section{Figure Captions}

Figure 1. Space -time paths in the EPR-Bohm experiment. The measurements are carried out simultaneously in this frame at $t=t_{i}$. (Arbitrary units).

Figure 2. The Horne-Shimony-Zeilinger (HSZ) experiment. Particle 1 encounters a beam-splitter $\left(H_{1}\right)$ at $x=-10$, whilst particle two encounters a beam splitter $\left(H_{2}\right)$ at $x=10$ (arbitrary units).

Figure 3. Space-time paths in the HSZ experiment in the $\Sigma$ frame. "ardt" labels the exit beam composed of the reflected part of packet $a(1)$ and the transmitted part of packet $d(1)$. The other labels have similar interpretation.

Figure 4. $\left|\Psi\left(z^{(1)}, t_{\mu} ; z^{(2)}, t_{\lambda}\right)\right|^{2}$ in the reduced configuration space $\left(z^{(1)}, z^{(2)}\right)$ shown with indicative trajectories for the case in which $\chi-\phi=0$ for scenario 1 in which the trajectories are initiated in positions for which $t^{(1)}=t_{\mu}$ and $t^{(2)}=t_{\lambda}$.

Figure 5. $\left|\Psi\left(z^{(1)}, t_{\lambda} ; z^{(2)}, t_{\mu}\right)\right|^{2}$ in the reduced configuration space $\left(z^{(1)}, z^{(2)}\right)$ shown with indicative trajectories for the case in which $\chi-\phi=0$ for scenario 2 in which the trajectories are initiated in positions for which $t^{(1)}=t_{\lambda}$ and $t^{(2)}=t_{\mu}$. 












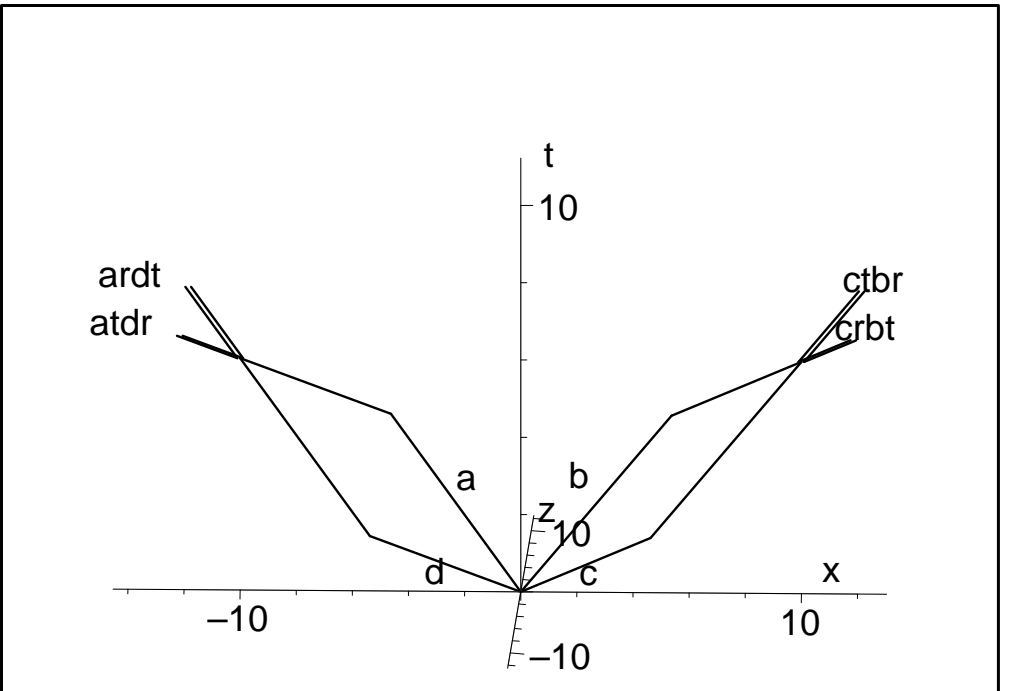




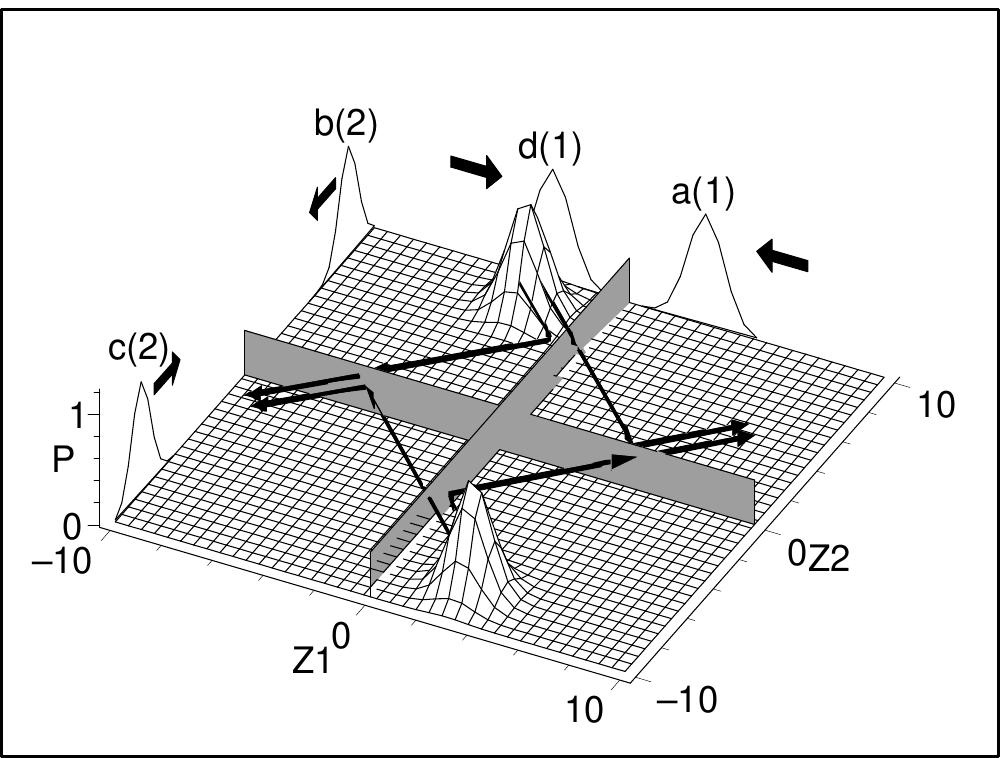




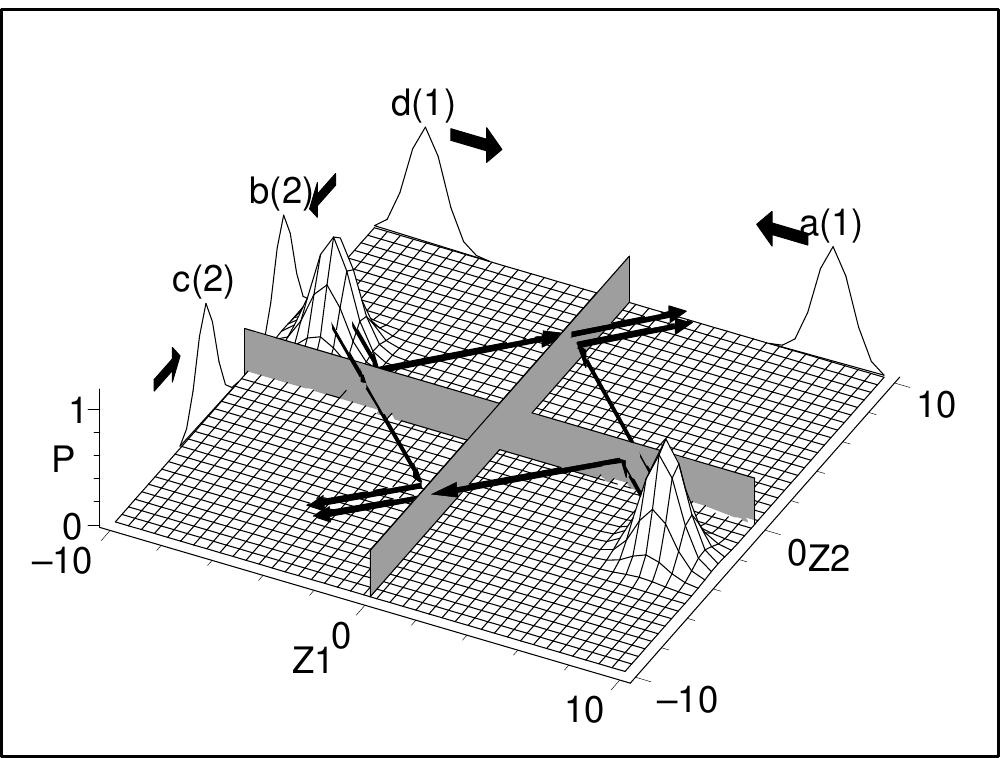

